\title{
Nonsurgical, nonorthotic treatment of occipital plagiocephaly: what is the natural history of the misshapen neonatal head?
}

\section{S. David Moss, M.D.}

\section{Phoenix Children's Hospital, Phoenix, Arizona}

Plagiocephaly has recently received increased attention in pediatric neurosurgical circles and its treatment has been controversial. Lack of uniformity persists in the treatment of neonates with occipital plagiocephaly. Plagiocephaly resulting from synostosis is rare. Many patients with nonsynostotic plagiocephaly have been treated surgically. Cranial remodeling orthotic devices (such as headbands and helmets) have also been reported to correct nonsynostotic plagiocephaly. The cost of orthotic treatment has risen and its validity has been contested by many third-party insurance payers. The effectiveness of orthotic treatment has not been adequately compared to the natural history of nonsynostotic plagiocephaly. The first phase of a natural history study was initiated in June 1995 at Phoenix Children's Hospital. All new patients referred with a diagnosis of plagiocephaly were categorized into two groups: those with mild-to-moderate asymmetry and those with moderate-to-severe asymmetry. Categories were determined by cephalic measurements. The patients with moderate-to-severe asymmetry were offered orthotic treatment with a cranial remodeling band. Those patients with mild-to-moderate asymmetry were observed with repeated measurements. Data in patients left untreated and followed for natural history were compared to our data published in 1994 for those treated with external cranial orthosis. Our published data in 47 patients treated with a headband during the period from 1988 to 1993 indicated that cranial vault asymmetry improved from a mean of $8.9 \mathrm{~mm}$ to $4 \mathrm{~mm}$ over an average treatment period of 4.3 months beginning when the average age of the patient was 5.9 months. Seventy-two neonates treated with neck stretching exercises and repositioning of the head without surgery or orthotic devices showed improvement in cranial vault asymmetry from $10.6 \mathrm{~mm}$ to $5.5 \mathrm{~mm}$ over an average treatment period of 4.5 months beginning when the average age of the patient was 4.5 months. This study indicates that neonates with mild-to-m oderate asymmetry who are less than 6 months of age and who are treated with physiotherapy and repositioning have similar improvement in cranial vault asymmetry as those treated with orthotic devices. The second phase of our study deals with neonates who have moderate-to-severe plagiocephaly.

\section{Key Words * plagiocephaly * synostosis * torticollis * headband}

The frequency of neonates presenting with occipital plagiocephaly has increased in most pediatric neurosurgical practices because of recommendations from the American Association of Pediatrics indicating the superiority of the supine position in preventing sudden infant death syndrome. Controversy exists regarding occipital plagiocephaly.[2,3,8,9] Plagiocephaly may result from either unilateral coronal or lambdoid synostosis. When it results from coronal synostosis, the occipital deformity is secondary, 
minimal, and seldom requires any treatment after correction of the frontoorbital deformity. Synostosis of the lambdoid suture is rare indeed. The author has performed only two such operations in the past 5 years in more than 100 craniofacial surgeries for synostosis. Surgical treatment for occipital plagiocephaly has continued to be contested but is currently applied in many centers in the United States.

Plagiocephaly occurs most frequently because of positional deformity, either in the prenatal period or during the first few weeks after birth. At times a child may show asymmetry of the cranium at birth due to positioning in the uterus or birth canal.[6] These neonates usually have an associated torticollis evidenced by preference in head turning to one side and/or head tilt to one side. Many of these children will also display a "windswept" appearance of the cranium to one side in addition to occipital flattening and cranial vault asymmetry. This initial plagiocephalic deformity is then worsened by gravitational forces as the child continues to rest the head on one side. The cranium itself tends to roll to the flat side, much like a ball that is flat on one side and always comes to rest on its flat surface. Children may develop occipital flattening after birth by repeated positioning on the same side of the occiput. This seems to be the most prevalent cause. Secondary deformity of the cranial vault then follows, which results in asymmetry of the face and ear position. This secondary deformity results from the same changes in shape to a parallelogram as it leans to one side (Fig. 1).

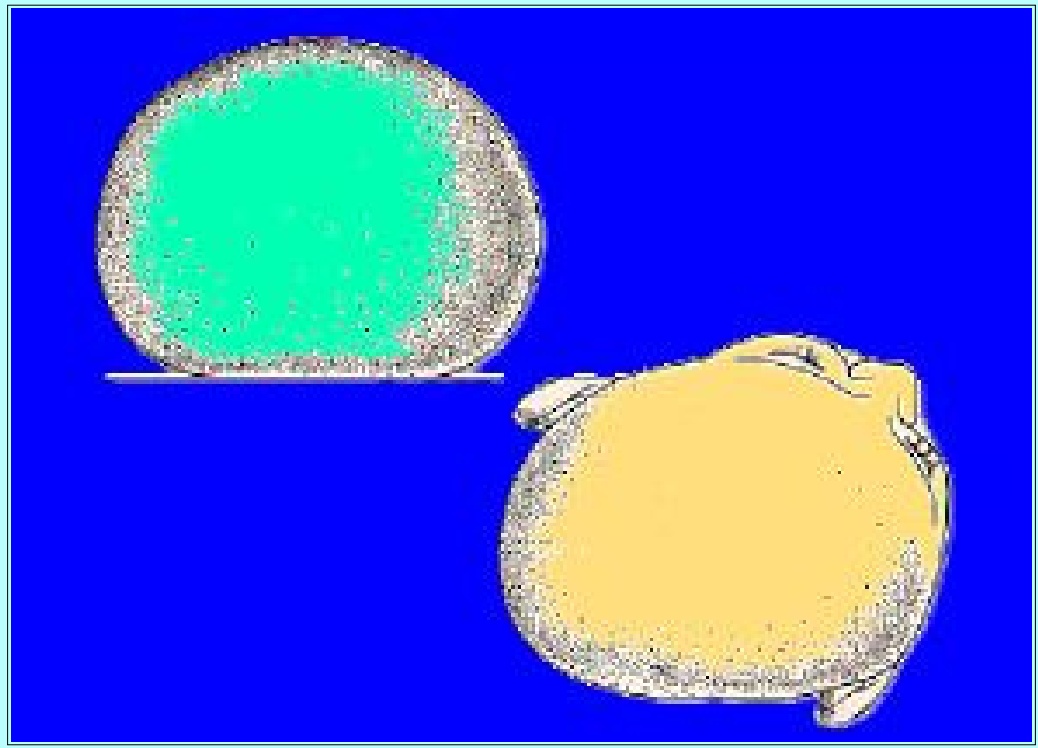

Fig. 1. Illustration showing cranial vault asymmetry due to gravitational forces. The picture on the left is a water-filled balloon placed on a flat table surface. The bottom becomes flat, as does the top somewhat and the sides bulge outward. The picture on the right shows the same effects on the cranium of a neonate, which results in asymmetry of the face and ear position. Note the parallelographic changes.

The diagnosis of synostosis can be excluded by examination on simple skull radiographs or computerized tomography (CT) scans.[1,4] One must be cautious to avoid diagnosing synostosis on the basis of sclerotic margins of the lambdoid suture. When the occiput is flattened due to positional plagiocephaly, the lambdoid suture is no longer bowed outward and at times can become flattened or indented. This may change the bone activity or appearance on each side of the suture resulting in the increased radiographic density seen along the suture, in spite of a clearly open suture line. This change may also reflect attempts at premature closure of the suture due to the plagiocephalic deformity. Whatever the cause, we have seen no difference in the response to nonsurgical treatment in patients with sclerotic lambdoid suture margins compared to those without this radiographic finding. 


\section{CLINICAL MATERIAL AND METHODS}

Beginning in June 1995, all children referred for treatment of potential plagiocephaly were evaluated by skull radiography or CT scanning to exclude synostosis. Children with synostosis were treated surgically; the type of correction depended on the kind of synostosis encountered. No incidence of lambdoid synostosis was found and no surgery was performed to correct occipital plagiocephaly. Perinatal histories were obtained to ascertain any prenatal positioning that may have been noted on prenatal examinations. Positioning of the neonate following delivery as well as a history of torticollis were also noted. Cervical spine radiographs were obtained in cases displaying significant torticollis. No vertebral deformity was found in any patient.

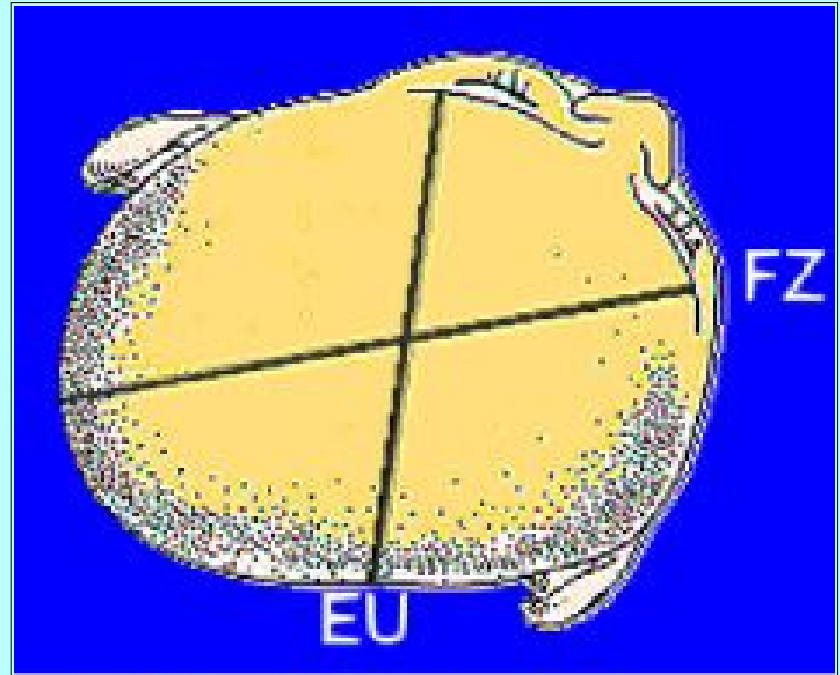

\section{Tragus}

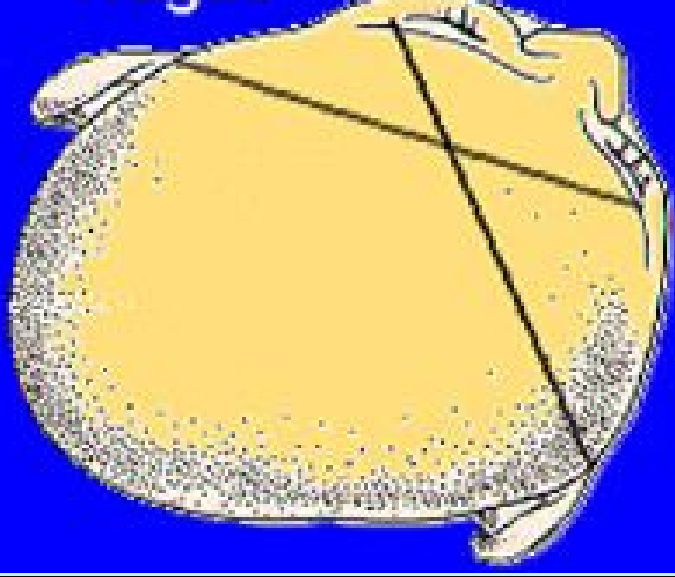

Fig. 2. Illustration showing cranial vault asymmetry (CVA). (CVA = FZ - EU Cross-Diagonal [mm]). The EU (eurion) has been taken at the point of largest measurement obtainable by placing the calipers on the FZ (frontozygomatic) point and moving the other arm of the calipers over the EU area. (Frontoorbital asymmetry $=\mathrm{FZ}-$ Tragus.)

Seventy-two consecutive children were evaluated using cephalic measurements to indicate cranial vault asymmetry as well as facial asymmetry (Fig. 2). These measurements are a subset of the measurements made during the study of outcomes for patients treated with cranial remodeling headbands.[10] The children were categorized into two groups: those whose measurements were $12 \mathrm{~mm}$ or less were designated as having mild-to-moderate asymmetry and those whose measurements were greater that $12 \mathrm{~mm}$ were designated as having moderate-to-severe asymmetry.

FZ Treatment with a cranial remodeling headband was offered for those children with greater than $12 \mathrm{~mm}$ asymmetry. If treatment was declined or if it was denied by their third-party insurance carrier, they were followed in the natural history group of patients. Six patients with measurements in the mild-to-moderate category were treated with a headband and thus were excluded from the natural history data. One patient failed to show improvement and necessitated treatment with a headband. Data obtained in that patient are included up to the point that the headband was applied.

Parents were encouraged to engage their children in neck stretching exercises frequently during the day and to place the child half supine with a towel or blanket roll behind the shoulder to position the occiput away from the flat side. The room was arranged and child placed to require the head to turn away from the flat side to see the parents and others in the room.

Patients were followed until both the physician and the family were comfortable with the results and were confident that the improvement would continue. This generally entailed the children being able to roll over, sit, crawl, or stand, thus spending minimal time on their backs. 


\section{RESULTS}

Seventy-two patients were followed for an average of 4.5 months and measured at 6-week to 2-month intervals. The average age of the patient at presentation and first cranial measurement was 6.6 months; the average age at completion of observation was 11.1 months. The mean asymmetry of the cranial vault measured $10.6 \mathrm{~mm}$ (range 4-17 mm). The mean measurement at the end of follow up was $5.5 \mathrm{~mm}$ (range $1-11 \mathrm{~mm}$ ). One patient worsened from 5 to $6 \mathrm{~mm}$ and was treated with a cranial remodeling band. It is of interest to note that following the headband treatment, the asymmetry remained at $5 \mathrm{~mm}$ in that patient after 4 months of treatment.

These results were compared to those published in 1993 in the same clinic setting and are shown in Table 1 .

\begin{tabular}{|ccc|}
\hline \multicolumn{3}{c|}{ TABLE 1 } \\
DEMOGRAPHICS IN 72 PATIENTS OBSERVED OR TREATED FOR PLAGIOCEPHALY \\
\hline \hline & Treated WI & Observedf or \\
Hariatle & Headtand & Natural History \\
\hline average age at presentation & $5.9 \mathrm{mos}$ & $6.6 \mathrm{mos}$ \\
duration of observation & $4.3 \mathrm{mos}$ & $4.5 \mathrm{mos}$ \\
initial asymmetry & $8.9 \mathrm{~mm}$ & $10.6 \mathrm{~mm}$ \\
ending asymmetry & $4.0 \mathrm{~mm}$ & $5.5 \mathrm{~mm}$ \\
\hline
\end{tabular}

\section{DISCUSSION}

Occipital symmetry has been restored by surgical reconstruction in occipital plagiocephaly thought to be due to synostosis of the lambdoid suture.[9] External orthotic treatment has been effective in restoring asymmetry in nonsynostotic occipital plagiocephaly.[2,8,10]

The results from Phase 1 of this study help to identify the outcomes in nonmedical intervention in benign positional or gravitational molding of the neonatal head. It is likely that nonsynostotic occipital plagiocephaly has existed throughout the centuries. It is seen somewhat more frequently now that we have focused our attention on the matter and because more children are maintained in the supine position for longer periods of time at early ages due to recent recommendations for preventing sudden infant death syndrome.[7] One needs only to examine the heads in the adult population to realize that the number with appreciable asymmetry is far less in this age range than in the neonatal population. One could deduce that the natural remodeling process of the human head must correct for many of the deformities seen in childhood. This author's grandmothers and a great-grandmother indicated that they believed it to be routine for mothers to reposition babies to obtain and maintain roundness of the head long before our current epidemic of flattened heads. Repositioning has been shown to be effective in restoring symmetry to the cranium.[5]

The cost of treatment using external orthotic devices has risen because of the expense incurred by companies marketing many of these devices. The cost may even rival that of many neurosurgical procedures such as the common ventriculoperitoneal shunt revision. Third-party insurance payers are skeptical about the value of these devices and are reluctant at times to reimburse for them. Neurosurgeons are also becoming more cost conscious and cautious about how they choose to spend neurosurgical resources. 
The results obtained in Phase 1 of this study strongly indicate that repositioning neonates with occipital plagiocephaly may produce improvement in cranial vault asymmetry very similar to that reported with external orthotic treatment. Continued vigilance is necessary to identify the patients who may not improve with repositioning and stretching exercises; rather, these patients may benefit from orthotic treatment. Phase 2 of this study will focus on identifying the outcomes in patients in the moderate-to-severe category who have cranial vault asymmetry measuring greater than $12 \mathrm{~mm}$.

\section{References}

1. Carmel PW, Luken MG III, Ascherl GF Jr: Craniosynostosis: computed tomographic evaluation of the skull base and calvarial deformities and associated intracranial changes. Neurosurgery 9:366-372, 1981

2. Clarren, SK: Plagiocephaly and torticollis: etiology, natural history, and helmet treatment. J Pediat 98:92-95, 1982

3. Dias MS, Klein DM: Occipital plagiocephaly: deformation or lambdoid synostosis? Pediatr Neurosurg 24:69-73, 1996

4. Furuya Y, Edwards MSB, Alpers CE, et al: Computerized tomography of cranial sutures. Part 1: Comparison of suture anatomy in children and adults. J Neurosurg 61:53-58, 1984

5. Hellbusch JL, Hellbusch LC, Bruneteau RJ: Active counter-positioning treatment of deformational occipital plagiocepaly. Nebraska Med J 80:344-349, 1995

6. Higginbottom MC, Jones KL, James HE: Intrauterine constraint and craniosynostosis. Neurosurgery 6:39-44, 1980

7. Kane AA, Mitchell LE, Craven KP, et al: Observations on a recent increase in plagiocephaly without synostosis. Pediatrics 97:877-885, 1996

8. Pattisapu JV, Walker ML, Myers GG, et al: Use of helmets in positional molding, in Marlin AE (ed): Concepts of Pediatric Neurosurgery. Basel: Karger, 1989, Vol. 9, pp 178-184

9. Persing JA, Deleshaw JB, Jane JA, et al: Lambdoid synostosis: surgical considerations. Plast Reconstr Surg 81:852-860, 1988

10. Ripley CE, Pomatto J, Beals SP, et al: Treatment of positional plagiocephaly with dynamic orthotic cranioplasty. J Craniofac Surg 5:150-159, 1994

Manuscript received January 14, 1997.

Accepted in final form January 20, 1997.

Address reprint requests to: S. David Moss, M.D., Phoenix Children's Hospital, 909 East Brill Street, Phoenix, Arizona 85006.

Click here to view commentary on this article. 